\title{
Method of Double Averaging for Modeling PFC Switching Converters
}

\author{
S. C. Wong and C. K. Tse \\ Dept. of Electronic \& Information Engrg. \\ Hong Kong Polytechnic University, Hong Kong
}

\begin{abstract}
This paper describes the modeling of power-factorcorrection converters under average-current-mode control. The approach employs a double averaging procedure, which first applies the usual averaging over the switching period and subsequently applies averaging over the mains period. The resulting model, after two averaging steps, is nonlinear and capable of describing the low-frequency nonlinear dynamics of the system. The parameter ranges within which stable operation is guaranteed can be accurately and easily found using this model.

Keywords - Averaging, modeling, power-factor-correction switching converters.
\end{abstract}

\section{INTRODUCTION}

Power-factor-correction (PFC) boost stages are widely used in off-line switching power supplies. The basic configuration of a PFC boost stage consists of (i) an average-current-mode (ACM) control loop which "forces" the input current to follow the waveform of the input voltage; (ii) a slow output voltage feedback loop which controls the amplitude of the "shaped" input current according to the power demanded by the load. The aim of the control is to achieve a unity power factor at the input side and to maintain power balance while keeping the output voltage at a nearly constant level [1], [2]. Because the process is nonlinear, stability analyses based on linearized models are inaccurate and often provide stability information (e.g., parameter ranges for stable operation) which are not necessarily consistent with reality. In particular, low-frequency period-doubling phenomena in PFC boost preregulators have been reported by Orabi et al. [3], [4], [5]. Also, fast-scale instability has also been reported by Iu et al. [6]. In this paper we propose a modeling approach that preserves the lowfrequency nonlinear dynamics of the PFC boost converter and hence is capable of revealing the phenomena found in [3], [4], [5]. Essentially we apply averaging twice in succession, over the switching period and the mains period, to obtain a doubleaveraged model in the form of a system of simple continuoustime differential equations which can be readily analyzed and numerically simulated. Stability information generated from this model is accurate for the purpose of locating the ranges of parameters within which the PFC boost converter operates in its normally expected regime.

\section{The PFC Boost Converter Under Average CURRENT-Mode CONTROL}

The system under study is a boost converter under ACM control, as in typical PFC applications. As shown in Fig. 1, the input voltage is taken as a template for input current shaping. This templete is multiplied by a factor which effectively adjusts the amplitude of the input current. This amplitude control is derived from a nonlinear computational process involving a variable from the output feedback loop and the input voltage. To see how it works, we consider the basic

\author{
M. Orabi and T. Ninomiya \\ Dept. of Electrical \& Electronic Systems Engrg. \\ Kyushu University, Fukuoka, Japan
}

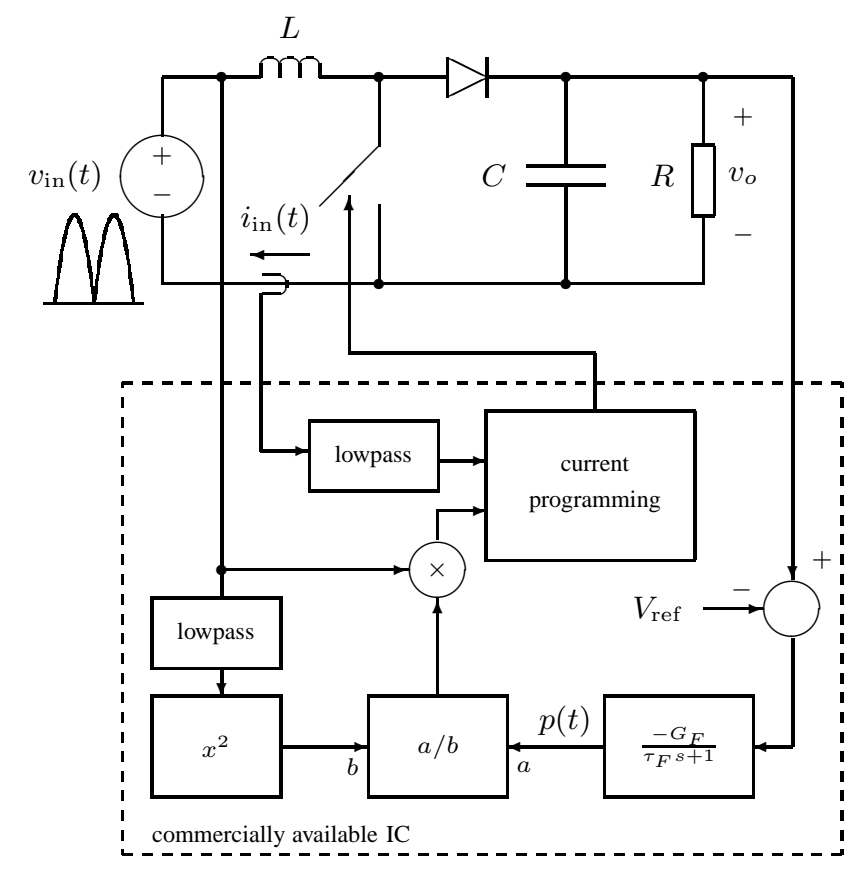

Fig. 1. Boost PFC converter under ACM control.

requirement of the input current waveform, which is to be "ideally shaped" as

$$
i_{\text {in }}(t)=\frac{p(t) \sqrt{2}}{V_{\text {in }}}\left|\sin \omega_{m} t\right|
$$

where $\omega_{m}$ is the mains angular frequency, $V_{\text {in }}$ is the rms input voltage, and $p(t)$ is the output power which is given by a feedback circuit. Furthermore, if we use the input voltage as a template for shaping the input current, the amplitude must include an additional denominator $V_{\text {in }}$, as depicted in Fig. 1, i.e.,

$$
i_{\text {in }}(t)=\frac{p(t)}{V_{\text {in }}^{2}} v_{\text {in }}(t)
$$

where $v_{\text {in }}(t)=\sqrt{2} V_{\text {in }}\left|\sin \omega_{m} t\right|$. As mentioned earlier, $p(t)$ is a feedback variable, which is derived from the output voltage. Obviously, $p(t)$ should vary very slowly relative to the mains half-cycle so that the sinusoidal waveform of the input current can be maintained. In practice, a low-pass type of feedback is applied to keep the output voltage nearly fixed. The usual control equation for $p(t)$ in the frequency domain is

$$
\frac{P(s)}{V_{o}(s)}=\frac{-G_{F}}{\tau_{F} s+1}
$$

where $P(s)$ and $V_{o}(s)$ are the frequency-domain variables of $\Delta p(t)$ and $\Delta v_{o}(t)$, respectively, and $G_{F}$ and $1 / \tau_{F}$ are the dc gain and cut-off frequency of the feedback circuit, respectively. 


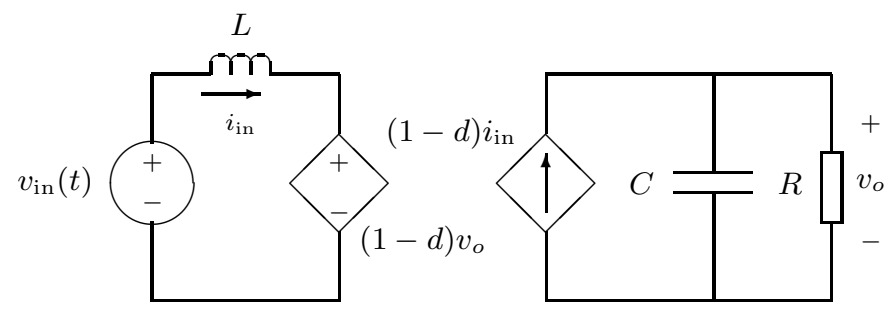

Fig. 2. First averaged model, where $d$ is implicitly defined by the ACM control.

Note that the equivalent time-domain equation is

$$
\tau_{F} \frac{d p(t)}{d t}+p(t)=-G_{F}\left(v_{o}-V_{\text {ref }}\right)
$$

where $V_{\text {ref }}$ is the reference output voltage. It is also worth mentioning that the overall $\mathrm{dc}$ gain in the loop must also include the factor $1 / V_{\text {in }}$ which is introduced by the divider at the input.

\section{Modeling By "Double Averaging"}

\section{A. First Averaging and Equation of Forced Oscillation}

For the purpose of studying the low-frequency dynamics, we may ignore the details within the switching cycle. This amounts to a standard averaging step that is to be taken over one switching period. The resulting model has appeared repeatedly in the literature of power electronics [7], [8]. We omit the details of the derivation here, but show the initial circuit model in Fig. 2. This model is incomplete because the duty cycle $d$ should be implicitly defined by the ACM control law. We first write down the Kirchhoff's law equations:

$$
\begin{aligned}
(1-d) v_{o} & =v_{\text {in }}(t)-L \frac{d i_{\text {in }}}{d t} \\
(1-d) i_{\text {in }} & =C \frac{d v_{o}}{d t}+\frac{v_{o}}{R}
\end{aligned}
$$

In the case of ACM control, the averaged inductor current has been programmed according to (1) or (2). From (5) and (6), and eliminating $d$, we get the following average power balance equation:

$$
\frac{C}{2} \frac{d v_{o}^{2}}{d t}=-\frac{v_{o}^{2}}{R}+i_{\text {in }} v_{\text {in }}-\frac{L}{2} \frac{d i_{\text {in }}^{2}}{d t} .
$$

For PFC applications, the energy storage in the inductor can be ignored. Thus, we get

$$
\begin{aligned}
& \frac{C}{2} \frac{d v_{o}^{2}}{d t}=-\frac{v_{o}^{2}}{R}+2 p(t) \sin ^{2} \omega_{m} t \\
& \frac{C}{2} \frac{d v_{o}^{2}}{d t}=-\frac{v_{o}^{2}}{R}+p(t)\left(1-\cos 2 \omega_{m} t\right)
\end{aligned}
$$

for $n \pi \leq \omega_{m} t<(n+1) \pi, n=0,1,2, \ldots$. It should be noted that $p(t)$ is connected with $v_{o}$ via the feedback circuit. Therefore, (8) is a continuous-time differential equation in $v_{o}$.

Thus, the system under study can be described by the set of equations consisting of (4) and (8). To analyze this system, we will make use of the fact that it describes a system of forced oscillation, where the variables are "driven" periodically, containing frequency components up to twice the mains frequency.

\section{B. Second Averaging}

We now consider further application of averaging to the system of equations under study, which consists of (4) and (8). Specifically, we take moving average over the mains period [9]. For any variable $a(t)$, we may assume that it takes the following form:

$$
a(t)=A_{0}+A_{1} \sin \left(\omega_{m} t-\theta_{1}(t)\right)+A_{2} \sin \left(2 \omega_{m} t+\theta_{2}(t)\right) .
$$

We define each component of the moving average of $a(t)$, denoted by $[a]_{k}$ for $k=0,1,2$, by

$$
[a]_{k}=\frac{\omega_{m}}{2 \pi} \int_{t-\frac{2 \pi}{\omega_{m}}}^{t} a(\tau) \exp \left(-j k \omega_{m} \tau\right) d \tau .
$$

For brevity and without confusion, we simply write $[a]_{k}$ as $a_{k}$. In other words, $a_{0}$ is the dc component of the moving average of $a(t), a_{1}$ is the component of the moving average of $a(t)$ at $\omega_{m}$, and $a_{2}$ is the component of the moving average of $a(t)$ at $2 \omega_{m}$. Thus, we may write

$$
\begin{aligned}
a(t)= & a_{0}(t)+a_{1}(t) e^{j \omega_{m} t}+\left[a_{1}(t) e^{j \omega_{m} t}\right]^{*} \\
& +a_{2}(t) e^{2 j \omega_{m} t}+\left[a_{2}(t) e^{2 j \omega_{m} t}\right]^{*}
\end{aligned}
$$

where superscript $*$ denotes complex conjugation. Upon expanding, we get

$$
\begin{aligned}
& a_{0}(t)=a_{0}=A_{0} \\
& a_{1}(t)=a_{1 r}+j a_{1 i}=-\frac{A_{1}}{2} \sin \theta_{1}-j \frac{A_{1}}{2} \cos \theta_{1} \\
& a_{2}(t)=a_{2 r}+j a_{2 i}=-\frac{A_{2}}{2} \sin \theta_{2}-j \frac{A_{2}}{2} \cos \theta_{2} .
\end{aligned}
$$

It is readily shown that the moving average of the timederivative of each component is related to the time-derivative of the component by

$$
\begin{aligned}
{\left[\frac{d a}{d t}\right]_{k} } & =\frac{\omega_{m}}{2 \pi} \int_{t-\frac{2 \pi}{\omega}}^{t} \frac{d a(\tau)}{d \tau} \exp \left(-j k \omega_{m} \tau\right) d \tau \\
& =\frac{d a_{k}(t)}{d t}+j k \omega_{m} a_{k}(t)
\end{aligned}
$$

where [ $]_{k}$ denotes consistently the $k$ th component of the moving average, with $k=0$ corresponding to the dc component, $k=1$ the $\omega_{m}$ component, and $k=2$ the $2 \omega_{m}$ component. Furthermore, the following relations are readily obtained:

$$
\begin{aligned}
{\left[a \cos 2 \omega_{m} t\right]_{k} } & =\frac{1}{2}\left(a_{k-2}+a_{k+2}\right) \\
{\left[a^{2}\right]_{0}=} & a_{0}^{2}+2\left|a_{1}\right|^{2}+2\left|a_{2}\right|^{2} \\
{\left[a^{2}\right]_{1}=} & 2 a_{0} a_{1}+2\left(a_{1 i} a_{2 i}+a_{1 r} a_{2 r}\right. \\
& \left.+j\left(a_{1 r} a_{2 i}-a_{1 i} a_{2 r}\right)\right) \\
{\left[a^{2}\right]_{2}=} & \left(a_{1}^{*}\right)^{2}+2 a_{0} a_{2} .
\end{aligned}
$$

\section{Derivation of the Double Averaged Model for PFC Boost Converter}

In this subsection we apply the foregoing averaging to (4) and (8). We expect to get three equations from each of (4) and (8), corresponding to the dc, $\omega_{m}$ and $2 \omega_{m}$ components. To avoid possible confusion due to mixup in subscripts, we define

$$
\left\{\begin{array}{l}
x=v_{o}(t) \\
y=p(t)
\end{array}\right.
$$


In the following, the use of subscripts will be consistent with the description of the second averaging in Section IIIB, i.e., subscript 0 for dc components, subscript 1 for mains frequency components, and subscript 2 for twice mains frequency components. Now, application of second averaging to (8) gives

$$
\begin{aligned}
\frac{C}{2} & \frac{d}{d t}\left(x_{0}^{2}+2 x_{1 r}^{2}+2 x_{1 i}^{2}+2 x_{2 r}^{2}+2 x_{2 r}^{2}\right) \\
+ & \frac{\left(x_{0}^{2}+2 x_{1 r}^{2}+2 x_{1 i}^{2}+2 x_{2 r}^{2}+2 x_{2 r}^{2}\right)}{R}=y_{0}-y_{2 r}
\end{aligned}
$$

$$
\begin{array}{r}
\frac{C}{2} \frac{d}{d t}\left(x_{0} x_{1}+x_{1 r} x_{2 r}+x_{1 i} x_{2 i}+j\left(x_{1 r} x_{2 i}-x_{1 i} x_{2 r}\right)\right) \\
+\left(\frac{j \omega_{m} C}{2}+\frac{1}{R}\right)\left(x_{0} x_{1}+x_{1 r} x_{2 r}+x_{1 i} x_{2 i}\right. \\
\left.+j\left(x_{1 r} x_{2 i}-x_{1 i} x_{2 r}\right)\right)=\frac{y_{1}}{2}-\frac{y_{-1}}{4}
\end{array}
$$

$$
\begin{aligned}
& \frac{C}{2} \frac{d}{d t}\left(2 x_{0} x_{2}+\left(x_{1}^{*}\right)^{2}\right) \\
& \quad+\left(j \omega_{m} C+\frac{1}{R}\right)\left(2 x_{0} x_{2}+\left(x_{1}^{*}\right)^{2}\right)=y_{2}-\frac{y_{0}}{2}
\end{aligned}
$$

Note that $y_{-1}$ is equal to $y_{1}^{*}$. Moreover, (4) is decomposed into

$$
\begin{aligned}
\tau_{F} \frac{d y_{0}}{d t}+y_{0} & =-G_{F}\left(x_{0}-V_{\text {ref }}\right) \\
\tau_{F} \frac{d y_{1}}{d t}+\left(j \omega_{m} \tau_{F}+1\right) y_{1} & =-G_{F} x_{1} \\
\tau_{F} \frac{d y_{2}}{d t}+\left(j 2 \omega_{m} \tau_{F}+1\right) y_{2} & =-G_{F} x_{2} .
\end{aligned}
$$

Thus, equations (21) through (26) are the double averaged equations for the PFC boost converter under study.

\section{Steady-State Solution}

To find the steady-state solution, we put all time-derivatives to zero, giving

$$
\begin{aligned}
x_{1} & =y_{1}=0 \\
x_{2 r} & =\left[\frac{V_{\text {ref }}-x_{0}}{2 \Delta}\right]\left[1+2 x_{0} \frac{1+4 \omega_{m}^{2} \tau_{F}^{2}}{G_{F} R}\right] \\
x_{2 i} & =\left[\frac{\omega_{m}\left(V_{\text {ref }}-x_{0}\right)}{\Delta}\right]\left[-\tau_{F}+x_{0} \frac{1+4 \omega_{m}^{2} \tau_{F}^{2}}{G_{F}}\right] \\
y_{2 r} & =\left[\frac{V_{\text {ref }}-x_{0}}{\Delta}\right]\left[G_{F}+2 x_{0} \frac{1-2 \omega_{m}^{2} C R \tau_{F}}{R}\right] \\
y_{2 i} & =\left[\frac{V_{\text {ref }}-x_{0}}{\Delta}\right]\left[\omega_{m} C+\frac{2 \omega_{m} \tau_{F}}{R}\right] x_{0}
\end{aligned}
$$

where

$$
\begin{aligned}
\Delta= & 1+\frac{4\left(1-2 \omega_{m}^{2} C R \tau_{F}\right) x_{0}}{G_{F} R} \\
& +\frac{4\left(1+\omega_{m}^{2} C^{2} R^{2}\right)\left(1+4 \omega_{m}^{2} \tau_{F}^{2}\right) x_{0}^{2}}{G_{F}^{2} R^{2}} .
\end{aligned}
$$

Putting the above equations in (21), and using (24), we get

$$
\begin{aligned}
& 8\left(1+\omega_{m}^{2} C^{2} R^{2}\right)\left(1+4 \omega_{m}^{2} \tau_{F}^{2}\right) x_{0}^{4} \\
& +8 G_{F} R\left[2-2 \omega_{m}^{2} C R \tau_{F}+4 \omega_{m}^{2} \tau_{F}^{2}\right. \\
& \left.+\omega_{m}^{2} C^{2} R^{2}\left(1+4 \omega_{m}^{2} \tau_{F}^{2}\right)\right] x_{0}^{3} \\
& +G_{F} R\left[-8 V_{\text {ref }}\left(1+\omega_{m}^{2} C^{2} R^{2}\right)\left(1+4 \omega_{m}^{2} \tau_{F}^{2}\right)\right.
\end{aligned}
$$

$$
\begin{aligned}
& \left.+G_{F} R\left(9-12 \omega_{m}^{2} C R \tau_{F}+4 \omega_{m}^{2} \tau_{F}^{2}\right)\right] x_{0}^{2} \\
& +G_{F}^{2} R^{2}\left[G_{F} R\right. \\
& \left.\quad+2 V_{\text {ref }}\left(-4+6 \omega_{m}^{2} C R \tau_{F}-4 \omega_{m}^{2} \tau_{F}^{2}\right)\right] x_{0} \\
& +G_{F}^{2} R^{2} V_{\text {ref }}\left[-G_{F} R+V_{\text {ref }}\left(1+4 \omega_{m}^{2} \tau_{F}^{2}\right)\right]=0
\end{aligned}
$$

from which $x_{0}$ can be solved numerically. Note that $x_{0}$ is the steady-state dc component of the output voltage, which is close to $V_{\text {ref }}$.

\section{Application of the Double Averaged Model to STABILITY ANALYSIS}

Our purpose in this section is to examine the stability of the system with respect to its operation at the forcing frequency $2 \omega_{m}$, which is twice the mains frequency. This is because the input is a rectified sine wave repeating at $2 \omega_{m}$, and all variables in the system are expected to repeat at this forcing frequency. Moreover, if the system is entrapped in an orbit of frequency $\omega_{m}$, i.e., at half the expected repetition frequency, ${ }^{1}$ the operation is considered undesirable for practical purposes since the device stresses would be drastically altered. In fact, such "period-doubling" has been observed experimentally when certain parameters are changed irrespective of the stability of the $2 \omega_{m}$-orbit [3], [4]. It is therefore of practical importance to locate the occurrence of period-doubling in the parameter space. In our double-averaged model, this is equivalent to finding the condition under which the system is entrapped into an $\omega_{m}$-orbit.

Our approach to solve this stability problem is to consider the loop gain of $x_{1}$ (or $y_{1}$ ) in our double averaged model. Thus, from (22) and (25), and the steady-state values solved from (28) through (31), putting the time derivatives to zero, we get the loop gain for $x_{1}$ as given in (34).

The system operates at the expected forcing frequency if it is not entrapped in the $\omega_{m}$-orbit, i.e., $T_{x 1}<1$. (It turns out that the condition $T_{x 1}>-1$ is irrelevant for practical sets of parameter values.) This condition is necessary for the system to operate in the expected regime. We may simplify (34) by putting $x_{0} \approx V_{\text {ref }}$, giving (35).

We note that the condition of $T_{x 1}<1$ is equivalent to (36). This means that normal operation of the circuit at $2 \omega_{m}$ can be disrupted by period-doubling if there are parametric variations that violates (36). This is sometimes considered as instability from an engineering viewpoint as the system fails to operate in the expected manner. In particular, we observe that

1) there is a lower limit of $v_{o} / V_{\text {in }}$ below which the system cannot operate normally at the forced frequency $2 \omega_{m}$ (i.e, operating range is limited);

2) this lower limit of $v_{o} / V_{\text {in }}$ increases as the load resistance increases (i.e., normal operation is disrupted by period-doubling as output power decreases to a certain threshold);

3) this lower limit of $v_{o} / V_{\text {in }}$ generally increases as $G_{F}$ increases (i.e., operating range is reduced as the feedback gain increases);

\footnotetext{
${ }^{1}$ In the literature of dynamical systems, such a change in operation is known as period doubling, and the transition from the normal operation to a double-period operation is typically a sudden transition. The usual term used to describe this transition is bifurcation [10]
} 


$$
\begin{aligned}
T_{x_{1}}= & \frac{G_{F} R}{2\left(4+\omega_{m}^{2} C^{2} R^{2}\right)\left(1+\omega_{m}^{2} \tau_{F}^{2}\right)\left(x_{0}^{2}-x_{2 r}^{2}-x_{2 i}^{2}\right)}\left\{\omega_{m} C R\left(\omega_{m} \tau_{F}\left(2 x_{0}-x_{2 r}\right)+x_{2 i}\right)\right. \\
& -2\left(\omega_{m} \tau_{F} x_{2 i}+2 x_{0}+x_{2 r}\right) \\
& \left.+\sqrt{\begin{array}{c}
-3\left(4+\omega_{m}^{2} C^{2} R^{2}\right)\left(1+\omega_{m}^{2} \tau_{F}^{2}\right)\left(x_{0}^{2}-x_{2 r}^{2}-x_{2 i}^{2}\right) \\
+\left[-2\left(\omega_{m} \tau_{F} x_{2 i}+2 x_{0}+x_{2 r}\right)+\omega_{m} C R\left(\omega_{m} \tau_{F}\left(2 x_{0}-x_{2 r}\right)+x_{2 i}\right)\right]^{2}
\end{array}}\right\} . \frac{3}{8} G_{F} R
\end{aligned} .
$$

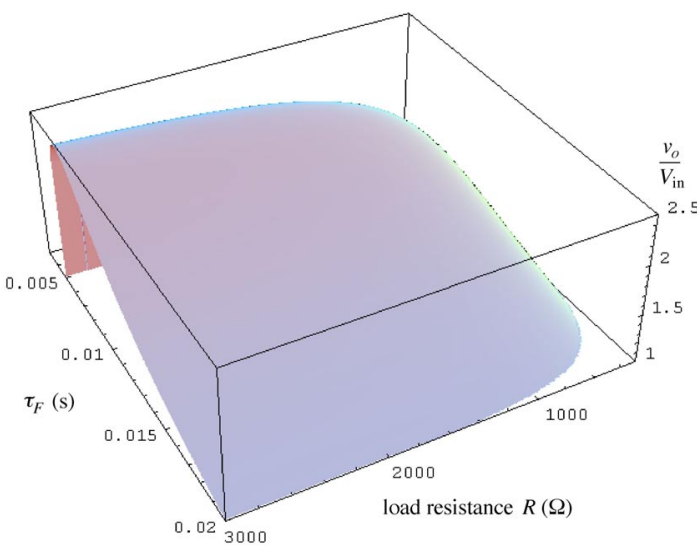

(a)

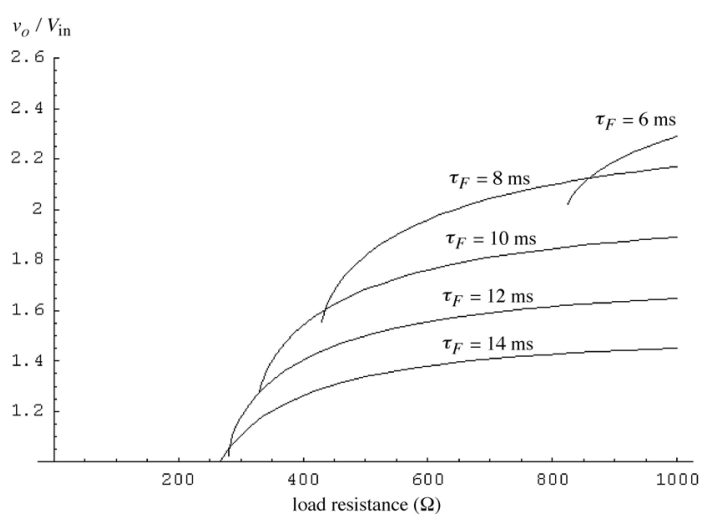

(c)

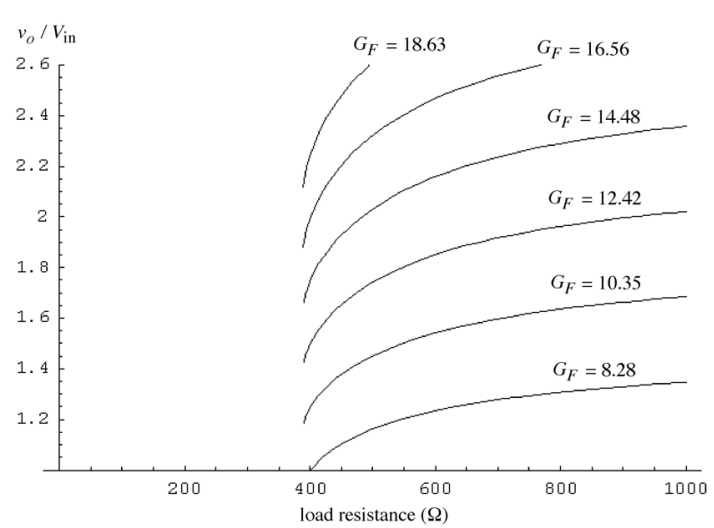

(b)

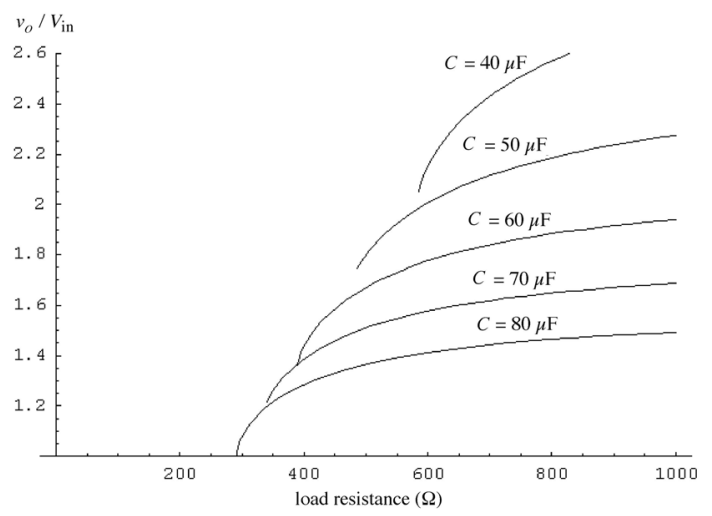

(d)

Fig. 3. Stability boundaries with (a) $G_{F}=12.8 \mathrm{~A}, C=60 \mu \mathrm{F}$ and $\omega_{m}=2 \pi(60) \mathrm{rad} / \mathrm{s} ;$ (b) $\tau_{F}=8.6 \mathrm{~ms}, C=60 \mu \mathrm{F}$ and $\omega_{m}=2 \pi(60) \mathrm{rad} / \mathrm{s} ;(\mathrm{c})$ $G_{F}=12.8 \mathrm{~A}, C=60 \mu \mathrm{F}$ and $\omega_{m}=2 \pi(60) \mathrm{rad} / \mathrm{s}$; (d) $\tau_{F}=8.6 \mathrm{~ms}, G_{F}=12.8 \mathrm{~A}$ and $\omega_{m}=2 \pi(60) \mathrm{rad} / \mathrm{s}$. Region above the boundary surface or curve corresponds to stable operating region. At the boundary, period-doubling occurs to disrupt normal operation. Region under the boundary surface or curve corresponds to "unstable operation" in the sense that normal operation cannot be maintained.

4) this lower limit of $v_{o} / V_{\text {in }}$ generally increases as $\tau_{F}$ decreases (i.e., operating range is reduced as the feedback time-constant decreases);

5) this lower limit of $v_{o} / V_{\text {in }}$ generally increases as the output capacitance decreases (i.e., operating range is reduced as output capacitance decreases).

\section{Graphical Representations of Stability BOUNDARIES}

To help visualize the above results, we use (36) to plot a few indicative boundary surface and curves. Our purpose 


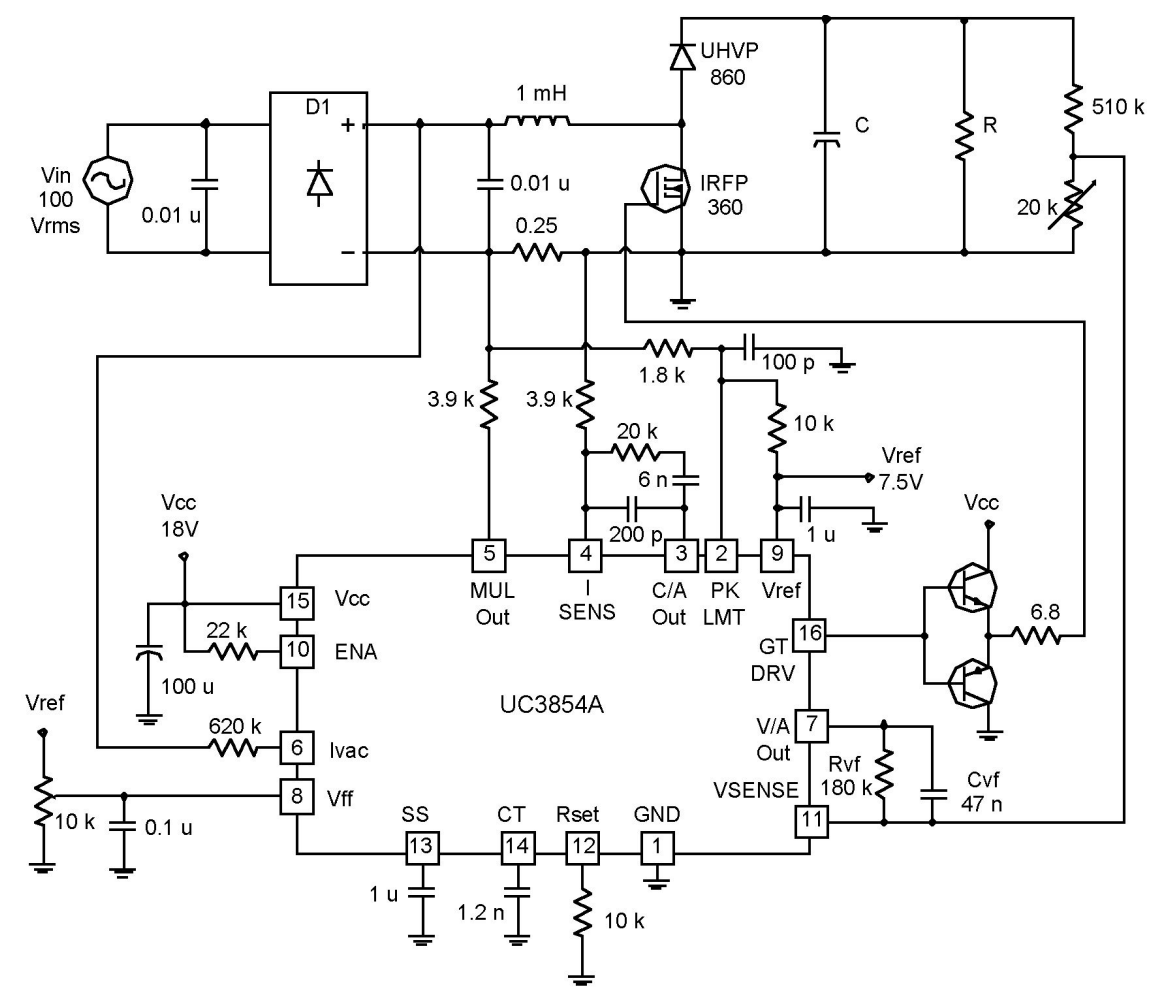

Fig. 4. Schematic of experimental power-factor-correction boost converter under average current-mode control.

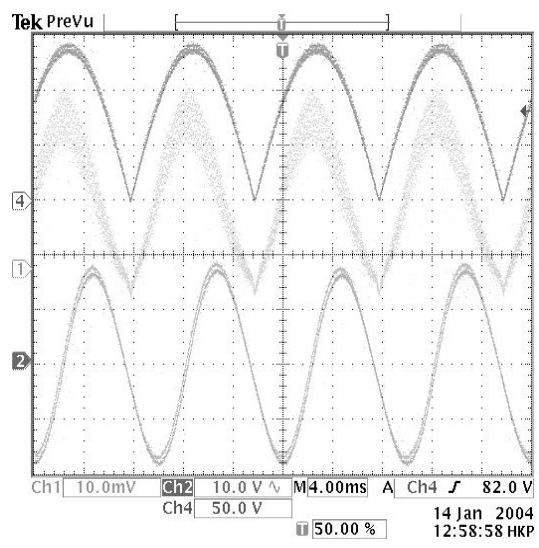

(a)

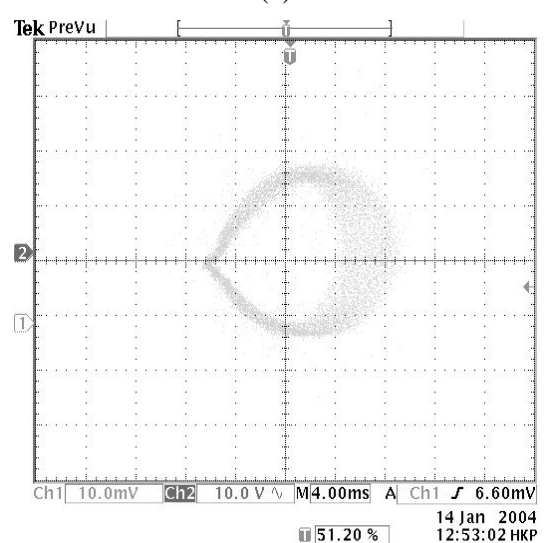

(d)

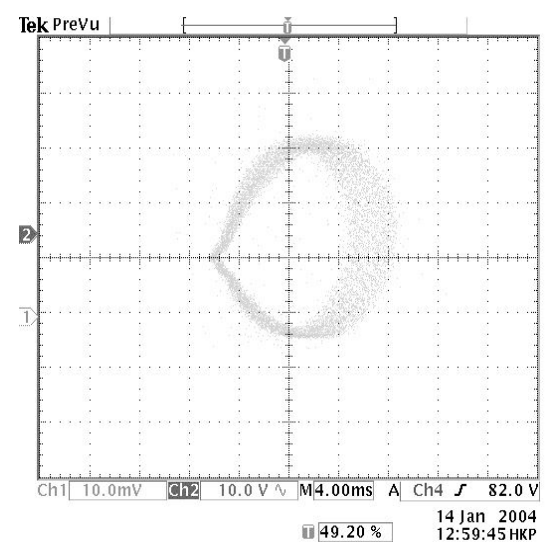

(b)

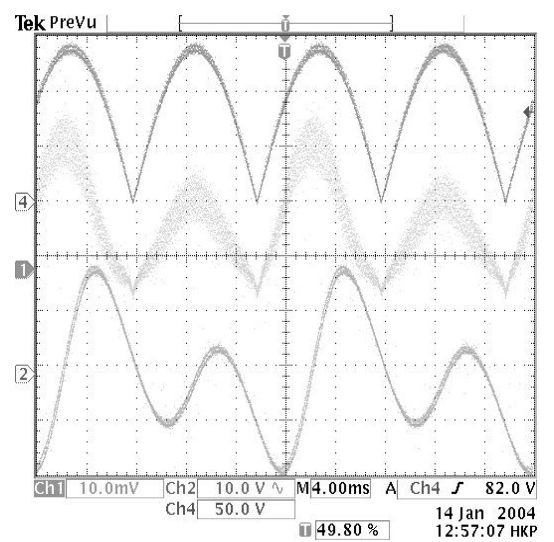

(e)

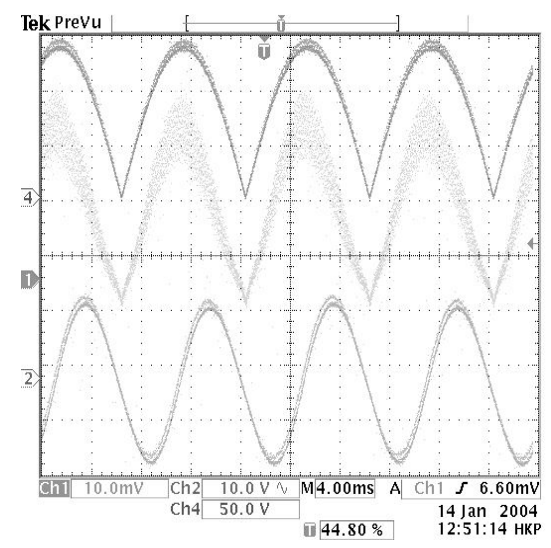

(c)

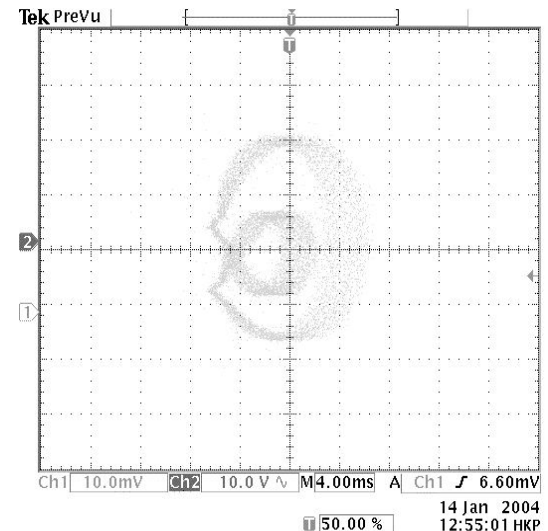

(f)

Fig. 5. Measured waveforms and phase portraits. (a)-(b) Normal operation with $R=454 \Omega$ and $V_{o}=297 \mathrm{~V}$; (c)-(d) normal operation with $R=645 \Omega$ and $V_{o}=360 \mathrm{~V}$; (e)-(f) period-doubled (unstable) operation with $R=645 \Omega$ and $V_{o}=297 \mathrm{~V}$. For all cases, $G_{F} \approx 20$ A, input voltage is $100 \mathrm{~V}$ rms, $\tau_{F}=8.46 \mathrm{~ms}$, and line frequency is $50 \mathrm{~Hz}$. For waveforms in (a), (c) and (e), upper trace is line voltage, middle trace is input current (1A/div) and lower trace is output voltage ripple. For phase portraits in (b), (d) and (f), x-axis is input current and y-axis is output voltage. 


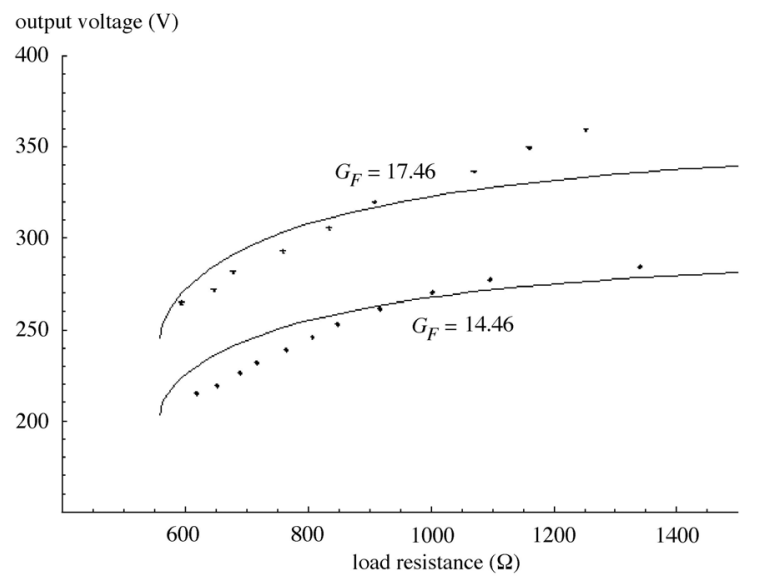

(a)

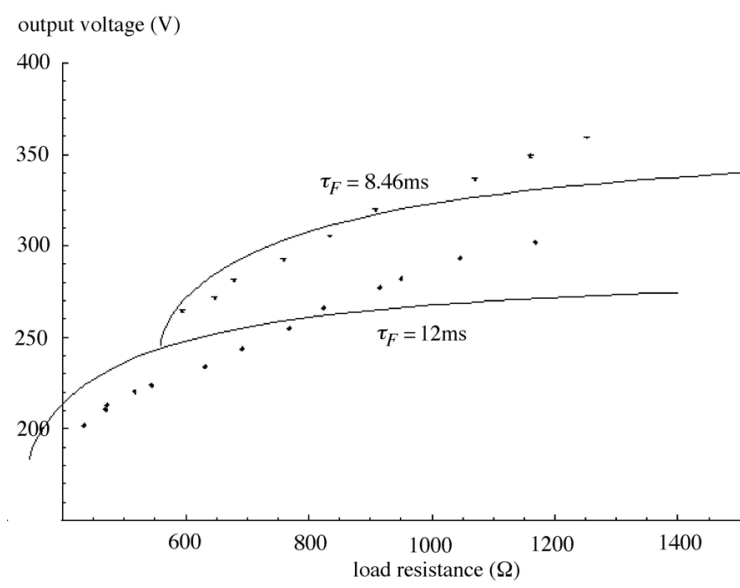

(b)

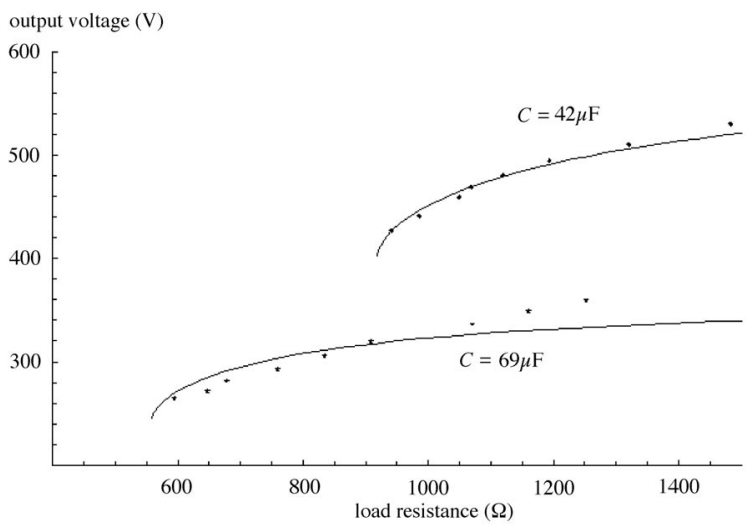

(c)

Fig. 6. Measured stability boundaries taken at $V_{\text {in }}=100 \mathrm{~V}(\mathrm{rms})$ and $50 \mathrm{~Hz}$ line frequency. Experimental data points are plotted as dots. Solid curves are from analytical expressions. (a) $C=69 \mu \mathrm{F}$ and $\tau_{F}=8.46 \mathrm{~ms}$; (b) $C=69 \mu \mathrm{F}$ and $G_{F}=17.46 \mathrm{~A} ;$ (c) $G_{F}=17.46 \mathrm{~A}$ and $\tau_{F}=8.46 \mathrm{~ms}$. Note that the y-axis would have been $v_{o} / V_{\text {in }}$ for better generality, as shown in our analytical study. However, for measurement convenience, we have fixed the input voltage in our experiments and varied the output voltage.

is to highlight the regions in the parameter space where normal operation is expected. Fig. 3 shows several operation boundaries corresponding to some specific sets of parameters. On the boundary surface or curves, period-doubling occurs and normal operation ceases. Region above the boundary corresponds to normal operation, where the system is driven periodically at twice the mains frequency.

\section{EXPERIMENTAL VERIFICATIONS}

An experimental PFC boost converter under average current mode control has been constructed for verification purposes. The complete schematic is shown in Fig. 4. The currentshaping control employs a standard 3854A integrated circuit controller. The line frequency is $50 \mathrm{~Hz}$. The input voltage is fixed at $100 \mathrm{~V}$ rms.

Experiments have been performed to verify the stability boundaries predicted in Sections V and VI

First, for a quick illustration, typical waveforms and phase portraits for normal operation and period-doubled (unstable) operation are shown in Fig. 5.

Our main objective is to locate the parameter points where period-doubling occurs. For convenience we replot the stability curves along with the experimental data in Fig. 6. We clearly see that the experimental data are in good agreement with the analytical results.

A few practical points are worth noting here:

1) One of the determining parameters is $v_{o} / V_{\mathrm{in}}$, as shown in our analytical study. However, for measurement convenience, we fix the input voltage in our experiments and vary the output voltage.

2) In our analytical model, the feedback loop is defined by two parameters, namely $G_{F}$ and $\tau_{F}$. In order to find the corresponding value of $G_{F}$ for the experimental circuit, we have to carefully identify all gains that may possibly contribute to $G_{F}$ since the model assumes no gain in the multiplier-divider block. In our experiment, we find this gain by direct measurement.

3) Our definition of $\tau_{F}$ is the real time constant. Thus, its reciprocal should be compared directly with the the angular mains frequency in $\mathrm{rad} / \mathrm{s}$ instead of the frequency in Hz. For instance, $\tau_{F}=1 / 2 \pi(50) \mathrm{s}=3.18 \mathrm{~ms}$ refers to a time constant equal to the period of a $50 \mathrm{~Hz}$ signal.

\section{CONCLUSION}

At present, the main technique for the analysis of power converters is based on averaging and subsequent small-signal 
linearization. Since power-factor-correction converters are subject to a time-varying input voltage and the current-shaping process is nonlinear, the results obtained from small-signal linear analysis have limited validity and often provide inaccurate stability information, as has been explained and demonstrated by Orabi et al. [3], [4], [5] with computer simulations and laboratory measurements. Nonlinear analytical models would be desirable for stability evaluation. This paper has introduced a method of double averaging for modeling power-factorcorrection converters, leading to nonlinear models from which closed-form stability conditions can be derived. This method is capable of revealing only the low-frequency stability of the circuit. For instability near the switching frequency, we need a different model, such as those based on discrete-time mapping [6], [11], for revealing the fast-scale instability.

\section{ACKNOWLEDGMENTS}

S. C. Wong and C. K. Tse gratefully acknowledge the support of Hong Kong Research Grants Council (CERG grant PolyU5241/03E) for carrying out this project.

M. Orabi and T. Ninomiya are supported by a research grant on the project "Control of Electromagnetic Environment in Low-Frequency Band Less Than $100 \mathrm{kHz}$ " of the future research promotion business in Japan, which has been provided by the Japan Society for the Promotion of Science.

\section{REFERENCES}

[1] L. H. Dixon Jr., "High power factor preregulator for off-line power supplies," Unitrode Switching Regulated Power Supply Design Manual, Paper 12, SEM700, 1990

[2] R. Redl, "Power-factor-correction in single-phase switching-mode power supplies - An overview," Int. J. Electron., vol. 77, no. 5, pp. 555-582, 1994.

[3] M. Orabi, T. Ninomiya, and C. Jin, "Nonlinear dynamics and stability analyses of boost power-factor-correction circuit," Proc. Int. Conf. Power Syst. Tech., pp. 600-605, 2002.

[4] M. Orabi, T. Ninomiya, and C. Jin, "A novel modeling of instability phenomena in PFC converter," Proc. IEEE Int. Telecom. Energy Conf., pp. 566-573, 2002.

[5] M. Orabi and T. Ninomiya, "Nonlinear dynamics of power-factorcorrection converter," IEEE Trans. Ind. Electron., vol. 50, no. 6, pp. 1116-1125, 2003.

[6] H. H. C. Iu, Y. Zhou, and C. K. Tse, "Fast-scale instability in a PFC boost converter under average current mode control," Int. J. Circuit Theory Appl., vol. 31, no. 6, pp. 611-624, 2003.

[7] R. D. Middlebrook and S. Cuk, "A general unified approach to modeling switching-converter power stages," IEEE Power Electron. Spec. Conf. Rec., pp. 18-34, 1976.

[8] C. K. Tse, Y. S. Lee, and W. C. So, "An approach to modelling DC-DC converters using graph theoretic concepts," Int. J. Circuit Theory Appl., vol. 21, no. 4, pp. 371-384, 1993.

[9] S. R. Sanders, J. M. Noworolski, X. Z. Liu, and G. C. Verghese, "Gerneralized averaging method for power electronic circuits," IEEE Trans. Power Electron., vol. 6, no. 2, pp. 251-259, 1991.

[10] C. K. Tse, Complex Behavior of Switching Power Converters, Boca Raton: CRC Press, 2003.

[11] C. K. Tse, O. Dranga and H. H. C. Iu, "Bifurcation analysis of a powerfactor-correction boost converter: uncovering fast-scale instability," Proc. IEEE Int. Symp. Circ. Syst., pp. III-312-315, 2003. 